\title{
LA INTENCIONALIDAD COMO IMPULSO FORMADOR: NOTAS PARA UNA LECTURA FENOMENOLÓGICA DEL ORIGEN CONJUNTO DEL ORGANISMO Y SU MUNDO CIRCUNDANTE
}

\author{
Diego Fernando Moreno Mancipe* \\ doi: 10.11144/Javeriana.uph37-75.imff
}

\section{RESUMEN}

Este artículo busca mostrar cómo una aproximación fenomenológica a la alteridad puede ser iluminada por un acercamiento al estudio del comportamiento de los animales. El vínculo entre etología y fenomenología, que solo es comprendido plenamente en su dimensión afectiva, se construye a través de una exploración en la que la intencionalidad (Intentionalität) fenomenológica aparece como impulso formador (Bildungstrieb) del organismo, su comportamiento y mundo circundante (Umwelt). Esto significa que la intencionalidad no constituye estructuras determinadas, sino procesos plásticos y dinámicos, capaces de vehicular tanto la génesis del organismo como la de su mundo. Entender la formación como un desarrollo epigenético permite advertir que la forma es un acontecimiento, el fruto de una potencia que va de lo general a lo particular, de las estructuras generales de la experiencia de una especie a la proliferación variopinta de perspectivas.

Palabras clave: fenomenología; intencionalidad; comportamiento; Umwelt; J. J. von Uexküll

Pontificia Universidad Javeriana, Bogotá, Colombia.

Correo electrónico: diegofmorenom@gmail.com

Para citar este artículo: Moreno Mancipe, D. F. (2020). La intencionalidad como impulso formador: notas para una lectura fenomenológica del origen conjunto del organismo y su mundo circundante. Universitas Philosophica, 37(75), 45-72. ISSN 0120-5323, ISSN en línea 2346-2426. doi: 10.11144/Javeriana.uph37-75.imff. 


\title{
INTENTIONALITY AS FORMATIVE IMPULSE: TOWARDS A PHENOMENOLOGICAL ACCOUNT OF THE GENESIS OF BIOLOGICAL ORGANISMS AND THEIR SURROUNDINGS
}

\begin{abstract}
This essay aims to show that a phenomenological account of animal behavior can shed light on an ethological approach to alterity, and vice versa. The interface between ethology and phenomenology -which, as it is argued, is intelligible only with reference to a shared dimension of affection- is framed by means of the phenomenological concept of intentionality (Intentionalität), which appears as a generative force (Bildungstrieb) of the organism, its behavior and surrounding world (Umwelt). This means that intentionality does not shape stable structures, but plastic and dynamic processes that bound both the genesis of an organism and its surrounding world. Formation is thus understood as an epigenetic development, which suggests that natural forms are the result of a movement that goes from the general to the particular, from the most broad and regular structures of experience, to the multifarious proliferation of perspectives.
\end{abstract}

Keywords: phenomenology; intentionality; behaviour; Umwelt; J. J. von Uexküll 


\section{Teleología y epigénesis}

En la "Analítica del juicio estético" de la Crítica del discernimiento, Immanuel Kant define fin o propósito como "el objeto de un concepto en la medida en que este se considera la causa de aquel (el fundamento real de su posibilidad)" (KU Ak. V220/B32). Este puede ser un artefacto o implemento, el resultado de un proceso teleológico, artístico o técnico, llevado a cabo por una razón exterior al objeto. Más adelante, Kant agrega que "un objeto [...] se denomina conforme a fines solo porque podemos explicar y concebir su posibilidad, únicamente, en la medida en que aceptamos como fundamento suyo una causalidad según fines" (KU Ak. V220/B33). Tenemos, entonces, un primer sentido en el que el fin apunta a cómo el objeto es producido; y un segundo sentido vinculado al ámbito del discernimiento, a cómo el objeto es conocido o comprendido. Es precisamente el segundo sentido del término el que Kant tiene en mente en la "Crítica del discernimiento teleológico", cuando se refiere a los organismos (Organisierte Wesen) como a "fines de la naturaleza" (Naturzwerk): los organismos pueden considerarse propósitos de la naturaleza pues, aunque no podamos determinar el proceso implícito de su génesis, su presencia parece exigir la consideración de cierta finalidad como condición de posibilidad e inteligibilidad de su existencia.

Para Kant es necesario considerar a los organismos desde una perspectiva teleológica, entenderlos como "fines de la naturaleza", ya que no es posible explicar su posibilidad a través de teorías mecánicas ${ }^{1}$. La forma de un ser vivo invita a apreciarlo "como si" (als ob) fuera el resultado de un plan. De hecho, las deficiencias del mecanicismo como modelo de comprensión de la vida se dan en dos niveles, según Kant: además de no responder a la pregunta por los principios de la organicidad, el mecanicismo tampoco da cuenta del orden ni de la regularidad

1 El término mecánico tiene varios sentidos y usos a lo largo de la obra crítica y precrítica de Kant: primero, en los Principios de la metafísica de la ciencia de la naturaleza de 1786, mecánicos son los efectos que los cuerpos en movimiento tienen unos sobre otros; segundo, en la Crítica del discernimiento, "Sobre el juicio teleológico", mecánico indica las relaciones físicas de la materia de acuerdo con las leyes del movimiento de Newton; tercero, también en este texto, mecánico significa lo contrario de teleológico, aquello que carece de finalidad como causa; y cuarto, en la Crítica de la razón práctica de 1788, mecánico se opone a teleológico cuando el segundo término implica liber$\operatorname{tad}$ (Ginsborg, 2014). 
de sus funciones vitales. De ahí que el concepto de propósito o fin esté asociado a una noción de legalidad específica en la "Crítica del discernimiento teleológico": referirse a un organismo como propósito implica reconocerlo como sujeto de leyes normativas que sustentan su autonomía anatómica y fisiológica. Sin embargo, a diferencia del objeto producido técnica o artísticamente, la forma del organismo es contingente, en tanto que no está determinada a desarrollar ninguna forma que preexista al organismo. La formación del organismo es libre porque este es fin en sí mismo, porque es una unidad de múltiples cuyo principio formal es inmanente en lugar de externo; un fin de la naturaleza es un objeto que se genera a sí mismo por naturaleza. Por ello, juzgar un objeto como producto natural, como fin de la naturaleza, significa apreciarlo como "causa y efecto de sí mismo" (KU Ak. V371/B286).

Con todo, el juicio teleológico es de carácter objetivo reflexionante, por ello no dice tanto del objeto en sí mismo como del ánimo del sujeto que juzga su forma. Kant afirma que "el concepto de los enlaces y formas de la naturaleza conforme a fines sí es, cuando menos, un principio adicional para colocar sus fenómenos bajo reglas allí donde no alcanzan las leyes de la causalidad con arreglo a su mero mecanicismo" (KU Ak. V360/B269). Pero esto no concede objetividad determinante a este principio secundario. El juicio teleológico aplicado a objetos naturales señala más un principio heurístico que un hecho científico, nos permite observar la naturaleza bajo principios investigativos, no explicativos.

Heredero de la filosofía de la naturaleza de Kant, y discípulo de científicos que abrazaron una comprensión teleológica de la vida como Joseph Müller, Karl Ernst von Baer y Hans Driesch, Jakob von Uexküll (1864-1944) estudió los organismos como vivientes activos y no como objetos. Su libro Ideas para una concepción biológica del mundo (1934) es un manifiesto en el que se declara la potencia objetiva de una concepción teleológica de la naturaleza limitada por Kant como reflexiva. Al igual que Kant, Uexküll reconoce dos tipos de objetos organizados de acuerdo con un plan: las máquinas y los seres vivos. En los dos ámbitos, "organización significa una unidad en la que las distintas partes se combinan en un todo a través de la agencia de una actividad común” (Uexküll, 1945, p. 105). La diferencia se encuentra en su génesis.

A diferencia del organismo, la máquina no se genera a sí misma. Al observar una máquina o un artefacto percibimos signos funcionales y genéticos que 
evidencian principios y fines externos a ella. La función que en el artefacto organiza las partes como un todo no es propia ni de las partes ni del conjunto. Por un lado, la forma del artefacto solo cobra sentido para nosotros en la medida en la que reconocemos su función; por el otro, desde la perspectiva del constructor, es manifiesto que el proceso de fabricación de la cosa es resultado de un agregado que conforma una unidad a partir de piezas desiguales que son enlazadas a través de acoplamientos. En toda máquina, función y génesis, unidad y productor, son distintos. Esto lleva a Uexküll a proponer una distinción entre fisiología o anatomía, el estudio de la forma (Gestalt) tal cual esta es percibida justo ahora, y morfología, el estudio de lo que Kant denominó proceso de formación (Bildung). En este sentido, no hay morfología de los artefactos pues, a diferencia de las artes y oficios en los que fuerzas centrípetas construyen la cosa juntando lo distinto desde afuera, "la morfología es una ciencia que inquiere sobre el modo centrífugo en el que se estructura el mosaico de células del que están compuestos los organismos" (Uexküll, 2017, p. 188).

La biología de Uexküll apela a unidades de estudio holísticas que permiten captar la relación entre el organismo en desarrollo y el espacio que le circunda. Su morfología se imbrica así con la etología, esto es, con la observación y descripción del comportamiento de los animales basado en el estudio de la manera en la que estos perciben el mundo. A fin de evitar una interpretación psicologista del mundo experimentado por los otros organismos, Uexküll opta por el estudio objetivo de los comportamientos y hábitos: el análisis de la estructura corporal de una especie animal asegura la posesión de los datos primarios para la comprensión del modo particular en el que una forma de vida extraña experimenta el mundo ${ }^{2}$.

Uexküll distingue el uso que hacemos de un artefacto y el comportamiento de un organismo. La forma en la que los organismos vivos actúan debe convencernos de que la regla de funcionamiento es responsable de su forma (la regla de

2 Precisamente el vínculo entre percepción y morfología descubre la diferencia específica entre la teleología de Kant y la de Uexküll. A pesar de sus múltiples referencias a Kant, el biólogo no sigue completamente la teleología del filósofo; para Uexküll no hay una fuerza teleológica más allá de la naturaleza, como sí aparece en la Crítica del discernimiento. Para Uexküll la conformidad a plan (Planmäßigkeit) que guía el desarrollo ontogenético del organismo no sigue ningún propósito objetivo antropologizado que, precisamente, solo el hombre podría percibir (Buchanan, 2008). 
digestión debe ser la causa de la aparición del sistema digestivo, por ejemplo). No obstante, metodológicamente debemos diferenciar dos momentos que, de cualquier manera, se encuentran correlacionados: la regla de génesis y la regla de funcionamiento.

Uexküll asume el modo en que opera la regla de génesis de manera contraria al preformismo y la teoría de la recapitulación ${ }^{3}$, teorías muy populares todavía en el siglo XIX y que admiten la existencia de una estructura anterior al cuerpo que debe ser satisfecha por el crecimiento material. Habría que esperar a las investigaciones embriológicas de Caspar Friederich Wolff y Johann Friederich Blumenbach para poner en cuestión tal presupuesto. Para Wolff, la ontogénesis es el proceso progresivo de coagulación (solidescibilitas) y solidificación de formas detalladas que, gracias a una fuerza esencial (vis essentialis), parte de una sustancia homogénea y termina en el desarrollo heterogéneo de órganos (Roe, 2003). Esta vis essentialis adquirió carácter semitrascendental con la epigénesis de Blumenbach: mientras que la fuerza esencial es una fuerza mecánica, la fuerza de organización embriológica es un factor de génesis teleológico. Para Blumenbach no existe una estructura anterior a la materia, sino un impulso formador (Bildungstrieb) que reglamenta el cumplimiento de una finalidad (Lenoir, 1989). Blumenbach distinguió su impulso formador de una fuerza formadora mecánica presente y perteneciente a la materia (Bildungskraft) gracias a su relación epistolar con Kant. Fue este último quien llamó su atención sobre la diferencia entre el tipo de legalidad determinante propia de las ciencias físicomecánicas y la legalidad libre a la que obedece la vida. Tiempo después, en el siglo XIX, Driesch demostró que el desarrollo morfológico del organismo no podía ser explicado acudiendo a una suerte de estructura invisible, etérea, organizada y altamente compleja oculta en el núcleo de las células: al dividir el embrión de un erizo de mar en dos obtuvo dos erizos de mar de la mitad del tamaño, no dos mitades diferentes de un erizo de mar.

3 El preformismo y la teoría de la recapitulación dominaron el clima científico hasta la aparición de la teoría de la epigénesis, a finales del siglo xviri. El preformismo niega la idea de una generación propiamente dicha, plantea que todo nuevo ser está ya contenido y encapsulado en el óvulo (ovulismo) o en el espermatozoide (espermatismo) antes de la fecundación; la teoría de la recapitulación de Haeckel plantea que la ontogenia recapitula la filogenia, es decir, el desarrollo de un organismo recapitula el proceso evolutivo de toda su especie (Laubrichler, 2008). 


\section{Melodía de formación y melodía de funcionamiento}

EN UNA DE LAS CARTAS dirigidas a su esposa, Uexküll escribe: "no sin premeditación probé que una caja de música nunca puede crear una melodía, sino que cada caja de música debe su existencia a una melodía" (Uexküll, 2014a, p. 98). La existencia de la caja de música es el producto de la melodía. Esta idea, recuperada de Karl Ernst von Baer, sirve para resaltar cómo la estructura del ser vivo es el producto del proceso de ontogénesis, no su causa. Lo común a la música y a los seres vivos es la aspiración a un fin como fuerza direccionada que reside al interior de los organismos. La embriogénesis es la construcción de un organismo con arreglo a un plan a partir de un impulso (Trieb) interior que, según la lectura que Uexküll hace de la teoría baeriana, expresa la tendencia hacia un objetivo o plan en términos temporales (Zielstrebigkeit) y espaciales (Planmäßigkeit) (Uexküll, 2010, p. 193).

Como ya mencionamos, Uexküll distingue dos melodías en toda ontogénesis: la primera es una melodía de origen, o melodía de formación, que rige la división celular y su determinación a partir del material protoplasmático; la segunda es una melodía funcional que rige el funcionamiento de los órganos y organismos ya desarrollados (Uexküll, 2014a, p. 100). Ahora bien, la coincidencia absoluta entre la regla de funcionamiento y la regla de formación es característica de los seres unicelulares. El cambio en la formación de órganos efectores de una ameba puede hacerse o deshacerse cada vez según la tarea percibida. La génesis de las propiedades de la ameba, forma, consistencia y adhesividad, son resultado de una tarea y se desfigura tan pronto como esta es completada. Siguiendo a Uexküll (2017), esto cambia cuando tratamos con organismos multicelulares: para ellos los órganos son primero formados y luego utilizados (p. 210). El primer momento es dominado por la regla de formación; el segundo, por la regla de

4 Uexküll aclara en Biología teórica que el paso de un régimen a otro se da justo en un "punto crítico" en el que el organismo se encuentra casi totalmente formado (Uexküll, 2017, p. 220). No obstante, Uexküll sabe bien que este acontecimiento no puede explicarse como un tránsito simple de un tipo de proceso a otro, por lo que se ve abocado a exponer en detalle la relación entre melodía de formación y melodía funcional. El modo en que esta imbricación es abordada por el biólogo ocupa el apartado 5 de este documento: "Melodía de formación". 
funcionamiento. El punto crítico en la ontogénesis de los organismos es alcanzado una vez la melodía de origen da paso a la melodía de funcionamiento.

El proceso de desarrollo, sin embargo, nunca cesa por completo. Así lo muestra la neotenia o conservación de características de inmadurez en individuos adultos. Más bien, la regla de funcionamiento altera el sentido de las fuerzas formadoras de los organismos: la fuerza centrífuga de la melodía de formación cede para encontrarse con las fuerzas centrípetas del exterior. "Si la estructura ha caído bajo el poder de la regla de funcionamiento, el conjunto de la región ambiental debe adaptarse a la creciente estructura e ir al paso con ella" (Uexküll, 2017, p. 221). A partir de entonces la melodía funcional tiende hilos de goma invisibles entre el entorno y el cuerpo del animal. No obstante, la melodía de funcionamiento es una regla regente, no derivada, dirá Uexküll (2017). En términos de Uexküll (2014a): "esta regla es -como regente del organismo que trabaja- el auténtico fin hacia el que tiende la melodía de formación” (p. 103). Si esto es así, entonces toda la ontogénesis tendría lugar con miras a realizar esta interconexión o correlación entre lo uno y lo otro, entre organismo y mundo. La melodía funcional consuma el ajustamiento en virtud del cual el organismo se relaciona con sus objetos en círculos funcionales (Funktionkreis). Así pues, el ajustamiento animal-mundo es el fin viviente hacia el que se dirige, desde el principio, toda formación. La conformidad a plan (Planmäßigkeit) junta como un imán aquello que se repelía, el sujeto y el objeto.

\section{El cuerpo vivo animal y la intencionalidad}

De ACUERdo CON CIERTA FISIOlogía MECANiCista a la que se opone la teoría biológica del mundo de Uexküll, un animal no es más que una selección determinada de herramientas de trabajo y de percepción sin intencionalidad, sin aquello que -a través de sus instrumentos u órganos- siente y hace. Quien, en contraste, considera que los órganos sensoriales y motrices llevan a cabo el obrar de los animales, descubre al maquinista (Machinesten) cuya intencionalidad es una con el cuerpo.

Uexküll considera que "cada célula del cuerpo es un sujeto independiente que posee reglas funcionales autónomas" (2017, p. 224). La célula tiene el poder de convertir los estímulos en presas de sus acciones. En virtud de lo anterior, toda 
célula goza de círculos funcionales propios - percepciones y acciones frente a tales percepciones- gracias a los que puede ensamblarse en un escenario más amplio, compuesto por otras células de su entorno. Ahora bien, la intencionalidad de los animales más complejos es distinta del tipo de subjetividad de las células o formas de vida básicas. La percepción y acción de un animal en su integridad puede atribuirse a la cooperación y ensamblaje de variados maquinistas celulares. No obstante, ¿cómo llega un animal a sentir y actuar como uno? ¿Qué hace posible que los órganos se entrelacen en una única percepción, en un único movimiento? En principio, Uexküll cree que basta un sistema nervioso central capaz de unificar las señales aisladas. Con todo, esta solución nos parece insatisfactoria por dejar intacto el problema de la síntesis del propio cuerpo y sus experiencias.

El sujeto de las investigaciones preetológicas de Uexküll no es el mismo sujeto u objeto de estudio de la psicología -el ego-; esa unidad producida por apercepción que, a su vez, se erige como principio de todo impulso motor. Atendiendo al tipo de investigación que le es legítimo llevar a cabo a la biología, para el naturalista el sujeto debe manifestarse como una unidad exterior, no interior. Este aspecto paradójico de la subjetividad también fue trabajado desde la fenomenología por Edmund Husserl en textos capitales como Ideas II o Meditaciones cartesianas. Husserl (2014, p. 204) denominó protopresencia a la percepción primigenia de un objeto que se encuentra ahí físicamente. Esto significa que la protopresencia es la cosa espacial (Dinge) que constituye un cuerpo físico (Körper). Con todo, el ser protopresentable no agota el ser de los objetos, ni siquiera su ser perceptible. Una dimensión nunca protopresentable es, por ejemplo, el ser animal (Animal), el ser animado que implica en efecto una subjetividad "copresentada". Así, nuestra experiencia del animal es la experiencia de una objetividad muy peculiar: al aparecer material del animal le pertenece la coaparición de la intencionalidad en sus actos. El comportamiento del organismo ofrece indicios indeterminados de lo que debemos suponer que son sus intenciones. El fenomenólogo se ocupa de las relaciones del otro con el mundo, no de los fenómenos tal y como estos son experimentados por él.

Uno de los problemas con el acercamiento husserliano a la alteridad es que se fundamenta en una fenomenología del cuerpo que deja al comportamiento en un nivel derivado. Ya que lo que pretendemos aquí es acercar la fenomenología a la biología descriptiva del comportamiento uexkülliana, debemos operar una 
variación metodológica: si lo primero que el fenomenólogo o el etólogo observan es un actuar que gradualmente llega a estabilizarse como el hábito de un único cuerpo vivo (Leib), entonces la fenomenología del cuerpo debe ser entendida como una fenomenología del comportamiento. La identidad del cuerpo percibido es aprehendida a través del decurso de los comportamientos y las observaciones; es un hacerse, no una estructura dada.

Lo anterior también exige superar la idea según la cual el cuerpo es el órgano de la voluntad. La expresión, el comportamiento, no es el acompañamiento exterior del pensamiento ni el accidente de un cuerpo que primero existe como objeto. Con la simple observación de que el gesto tiene un significado superamos el dualismo vulgar. Al observar el comportamiento animal no hay en la cabeza del etólogo un concepto de volición interior que posteriormente se traduce en acción. Más bien, el naturalista entiende de inmediato cómo el gesto mismo es portador de sentido. La vida anímica del animal no se traduce en el gesto, antes bien, se presenta y es una con él.

Como expuso Maurice Merleau-Ponty en La fenomenología de la percepción (1985), el mecanicismo y el intelectualismo fracasan en comprender la unidad del cuerpo fenomenal, del cuerpo que es experimentado como propio por un ser vivo. Mientras que el mecanicismo de tipo fisiológico cree encontrar en el sistema nervioso central el órgano de interconexión y coordinación de todos los demás sistemas, el intelectualismo, inclusive el fenomenológico trascendental, confía a una actividad de la conciencia la síntesis de las distintas sensaciones producidas en diferentes partes del cuerpo. Así, ambas posiciones esquivan la unidad anímico-corporal anterior a toda escisión objetiva o idealista. Empero, las diferentes partes del cuerpo, los órganos, no son simplemente coordinadas por un sistema físico o una actividad psíquica. Cuando el animal se dirige hacia una presa, las distintas tareas corporales se distribuyen por sí mismas entre los órganos, que se disponen de acuerdo con un sentido o propósito común. Las fracciones del cuerpo son conocidas por su valor funcional y su coordinación no es aprendida. El animal tiene una omnicomprensión de su propio cuerpo, inclusive de los órganos que jamás ha visto, sin necesidad de recurrir a la intervención de actividad intelectual alguna. La unidad del cuerpo animal se da de golpe y en situación, como un saber implícito de lo propio que no es necesario reanudar conscientemente. Es decir, la unidad del cuerpo fenomenal, del cuerpo 
que siente y actúa, no se realiza progresivamente de manera acumulativa, sino que ocurre de facto. La unidad del cuerpo es la condición de posibilidad, el horizonte de sentido, para utilizar una expresión fenomenológica, sobre el que cobra significado cualquier distinción sensorial y cualquier movimiento local.

Maurice Merleau-Ponty emplea la noción de esquema corporal para describir el modo en el que el cuerpo se da desde sí mismo como un sistema de equivalencia cinestésico que encarna una unidad dinámica en situación. El esquema corporal, término empleado en un principio por la fisiología, y luego por la Gestaltpsychologie, hace referencia al cuerpo como posesión indivisa de un todo que se opone a la idea de cuerpo como conglomerado de órganos yuxtapuestos. Para la fisiología, el esquema corporal no era más que una expresión útil para señalar el resumen de la experiencia corpórea con la que se buscaba designar el desarrollo temporal y gradual de la asociación entre órganos y sensaciones. Por su parte, para la Gestaltpsychologie, el término designa una toma de conciencia global de la postura intersensorial en el mundo que no es resultado de una asociación en el curso de la experiencia. Se trata de una forma (Gestalt) en el sentido de un "saber del propio cuerpo como término de conjunto y de la relación mutua de sus miembros y sus partes" (Bürger-Prinz \& Kalia, citados en Merleau-Ponty, 1985, p. 116n). Con todo, Merleau-Ponty expuso las limitaciones de afirmar el cuerpo como forma cuando esto significa un todo anterior a las partes, algo dado de antemano. Y es que también el esquema corporal obedece a un impulso formador. El sistema corporal es dinámico, no estático. El esquema corporal varía según mute la intencionalidad con la que el organismo se dirige a las tareas o acciones propuestas. Merleau-Ponty (1985) explicita el contraste entre los cuerpos objetivos y el cuerpo fenomenal sirviéndose de la siguiente comparación:

El sujeto situado frente a sus tijeras, su aguja y sus faenas familiares no tiene necesidad de buscar sus manos o sus dedos, puesto que no son objetos que hay que buscar en el espacio objetivo, huesos, músculos, nervios, sino potencias ya movilizadas por la percepción de las tijeras o de la aguja, la punta central de los "hilos intencionales" que lo vinculan con los objetos dados (1985, p. 123).

El maquinista animal es uno con sus órganos y sus movimientos porque para él estos no son herramientas, sino potencias para afectar y ser afectado. Cuando ejecuta un movimiento, el animal asume cierta situación de caza, alerta, huida, 
etc., que organiza y dispone el conjunto para actuar de un modo puntual. El cuerpo del animal es una forma dinámica que se distingue en su propia experiencia de aquello que no es él, de aquello a lo que atiende, debido a que está polarizado por sus tareas, que existe hacia ellas, que se recoge en sí mismo para alcanzar su objetivo (Merleau-Ponty, 1995). Entonces el esquema corpóreo indica que el cuerpo es del mundo. El animal experimenta el movimiento de su ser como postura vital en un escenario, no como una ilación de partes extra-partes.

Entendida desde la autoconciencia alcanzada téticamente, la fenomenología consideró que la conciencia de sí es constituida por cada uno inmanentemente, en una relación especular en la que cada sujeto se hace a la vez objeto de sí mismo. En las Meditaciones cartesianas Husserl señaló como primer estrato de la constitución de la primera esfera de propiedad de la conciencia la relación de desdoblamiento en la que el ego se constituye como tocante y tocado. La epoché temática gracias a la que logra hacer "abstracción de todas las efectuaciones constitutivas de la intencionalidad referida de modo inmediato o mediato a la subjetividad extraña” (Husserl, 2009b, p. 125) pone entre paréntesis todo sentido de objetividad extraña y recoge a la conciencia en sí misma. En este ejercicio, el primer cuerpo natural que la conciencia encontraría en el espacio que va más allá de su ámbito de inmanencia sería su propio cuerpo orgánico. La relación con un cuerpo extraño o ajeno al mío es simplemente un estrato fundado en esta experiencia originaria con nuestra propia corporalidad, y por lo mismo dependiente de ella. Pero tal delimitación de las potencialidades e intencionalidades del cuerpo vivo requiere, justamente, la suspensión de la naturalidad de las relaciones entre el ego encarnado, los otros y el mundo (Husserl, 2009b, p. 131). Si esto es así, para que el otro aparezca como algo más que un ornamento superfluo de nuestro mundo es menester que la experiencia de cada existencia no se reduzca a la autoconciencia, es decir, que permanezca y se comprenda a sí misma como ser natural.

Ahora bien, que el animal conoce la unidad de su propio cuerpo, aún sin tener autoconciencia de ello, es evidente por la forma en la que actúa frente a otros. Los animales que acechan o buscan escapar de otros se ocultan para no ser vistos. Recogerse, agacharse, resguardarse, sumergirse, agazaparse, detenerse, contener la respiración y esperar... todos estos, modos de escondite, vigilancia y emboscada, muestran que el animal actúa con un saber incorporado de su propia existencia en relación con lo otro. "Gracias a esta orientación del ser en relación 
consigo mismo, a esta polarización afectiva de todo contenido y de todo constituyente psíquico, [...] el ser conserva su identidad" (Simondon, 2015, p. 198). $\mathrm{El}$ animal se dirige a un mundo que le devuelve la mirada en una relación que podríamos llamar diádica. Ser-para-el-otro al revelarse u ocultarse, y ser-para-sí son dos estructuras cooriginaras, tanto de la existencia unitaria del animal, como de su experiencia del mundo, que desmienten toda constitución solipsista.

Los organismos se descubren unos a otros, y a sí mismos, como distintos del resto, en contexto, como coexistiendo en una naturaleza común. Hay un vínculo inquebrantable entre comportarse como uno y percibirse como diferente del otro que hay enfrente de sí. Algunos animales están abiertos a un mundo que les resulta, así, paradójico: pertenecen y se diferencian de él. En términos fenomenológicos, diríamos que con la constitución de la unidad orgánica del animal acontece la constitución de la unidad del mundo que se le aparece, del mundo exterior que se le contrapone (Gegenwelt), según Uexküll (2016).

\section{Hacia una comprensión fenomenológica del comportamiento}

LA SOLIDEZ DEL ENFOQUE BIOLÓGICO UEXKÜLLIANO frente a lo que este denomina "enfoque fisiológico" es apreciable en su verdadera magnitud gracias a los principios con que ambos se dedican a la observación de los ciclos vitales de los animales: para el fisiólogo, todo ser vivo es un objeto del mundo humano, mientras que para el biólogo el ser vivo es sobre todo un sujeto que habita su propio mundo (Uexküll, 2016). Podemos señalar como condición necesaria para la posesión de un mundo el que este siempre se abre para una subjetividad, para un maquinista. Planteada la pregunta èes la garrapata una máquina o un maquinista?, el fisiólogo responderá que la garrapata es poco más que una máquina compuesta de aparatos receptores y efectores interconectados por un mecanismo de control interno, un circuito de inputs y outputs basado exclusivamente en la necesidad causal del reflejo. En el arco reflejo del fisiólogo el maquinista está ausente. La descripción del mismo hecho varía significativamente asumiendo una perspectiva biológico-uexkülliana: los organismos no solo reaccionan a causas exteriores; antes que nada, se comportan, se dirigen hacia lo otro de sí. La constitución corporal de los animales destila los estímulos. "En esta perspectiva, la agencia del mundo exterior, del universo objetivo, desde ahora juega el rol del 
signo, en vez del de causa" (Merleau-Ponty, 1995, p. 226)5. Lo anterior significa que lo que llamamos estímulo no es recibido y transferido, sino percibido por un sujeto. No hay estímulo o sensación pura que no haya sido invitada o avivada por la percepción y acción del organismo.

Entre el mundo exterior (Welt) y el organismo viviente existe una imbricación que ordena, coordina, interpreta, discrimina y elabora. Por una parte, las señales perceptuales se unifican por fuera de los órganos perceptivos en unidades que devienen propiedades de los objetos; por la otra, las señales efectuales son asidas en unidades exteriores que reclaman cierta acción.

A manera de ilustración, podemos decir que cada sujeto animal aprisiona su objeto con los dos brazos de una tenaza: un brazo perceptual y uno efectual. Con el primero, le asigna al objeto un signo perceptual, mientras que con el segundo le asigna un signo efectual (Uexküll, 2016, p. 43).

Esto quiere decir que toda propiedad perceptivamente significativa influye sobre las propiedades efectualmente significativas operando un efecto que Uxküll llama transformador. El ciclo perceptivo-efectivo entre el animal y su objeto puede resumirse con el esquema del círculo funcional (Funktionkreis), concepto con el que el biólogo expresa la adaptación de uno a otro en un todo orgánico. El concepto central de la filosofía biológica uexkülliana, Umwelt o mundo circundante, abarca el todo compuesto por el sujeto y la totalidad de los objetos que a este le resultan significativos, es decir, la totalidad de los círculos funcionales actuales y posibles.

Una aproximación biológica radical al comportamiento y al mundo de los animales debe ir inclusive más allá. Es menester que la percepción y la acción que constituyen el círculo funcional sean entendidas como potencias intencionales, esto es, que la percepción y la efectuación de movimientos se muestren como dirigiéndose o volcándose hacia los objetos, más que como pasividad o reactividad $^{6}$. Si el animal puede oponer su ser a alguna otra cosa, si incluso puede

5 «Dans cette perspective, l'agencement du monde exterieur, l'univers objectif, joue desormais plutot Ie role de signe que celui de cause » (Merleau-Ponty, 1995, p. 226).

6 Vinciane Despret (2018) se ha encargado de problematizar el uso de los conceptos de reacción y reactividad tan populares en la literatura del comportamiento animal. Según esta filósofa belga, con el término reacción "los autores prolongan una concepción de un animal pasivo, totalmente 
saberse vivenciando y actuando frente a algo que le resulta extraño, es en virtud de que esta experiencia perceptivo-efectual está remitida a cierta trascendencia, a un más allá de sí mismo, que, no obstante, en tanto que experiencia, es constituido por él. Basta con observar la manera en la que ciertos animales anticipan o prevén sus movimientos para percatarse de que no todo movimiento animal es reactivo o concreto, que no todo movimiento responde a un estímulo efectivamente presente al modo del arco reflejo.

Para Merleau-Ponty (1985), el movimiento es experimentado como parte de una situación, como un eslabón de los acontecimientos. El animal, como el comediante del que habla el fenomenólogo francés, puede fantasear situaciones, como cuando se irrealiza en un juego que no confunde con una situación seria. El animal no es una máquina que responde condicionadamente a los estímulos del mundo, esto es claro por la forma como su cuerpo se separa de su situación concreta para respirar, moverse o luchar de la manera que la ficción le exige. El juego entre crías muestra cómo los animales pueden proyectar situaciones no actuales, solicitando comportamientos que fluyen desde un contexto virtual, como en la vida normal. Entonces, el animal prevé y la intención constituye para sí y para otros un espacio y un tiempo que no le es dado. Esta intencionalidad del movimiento abstracto es mejor entendida cuando es abordada como variación del ser total de un sujeto, y no como excepcionalidad que reta los angostos límites de la tipicidad. En general, podemos afirmar de la salud del organismo animal lo mismo que Merleau-Ponty (1985, p. 125) afirmó de la salud física y espiritual de un ser humano, esto es: un organismo saludable no dispone únicamente de su cuerpo cuando está implicado en un medio contextual concreto y respecto de tareas ya dadas en un oficio real, sino que, además, su cuerpo es correlato de posibilidades puras desprovistas de significación práctica, que está abierto a situaciones ficticias que puede escoger o que un experimentador puede proponerle.

determinado por causas que lo superan y sobre las cuales no tiene ningún dominio” (p. 152). Para Despret es evidente cómo esta comprensión del comportamiento animal es producto del trabajo con especímenes de laboratorio, esclavos de las escenas que los científicos fabrican para ellos. Es así como el "quehacer científico" hace desaparecer toda iniciativa. Si, por el contrario, se le concede al animal una perspectiva propia (p. 138), entonces es posible verlo participar activamente como actor de la puesta en escena, a la que responde según sus propios intereses y su intención de mostrarse de cierta manera frente a los otros. 
Hace parte del horizonte intencional de la vida salir de la esfera de la presencia del ser e introducirse en el no ser. En general, comenta Merleau-Ponty (1985), "el normal cuenta con lo posible que así adquiere, sin abandonar su lugar de posible, una especie de actualidad" (p. 126). Tanto el movimiento como la percepción tienen un fondo; cada movimiento y cada percepción es un momento de un contexto total único. El fondo no es una representación exterior al movimiento y a la percepción misma, es inmanente, los anima y mueve. Así, la distinción entre comportamiento abstracto y comportamiento concreto puede entenderse como una distinción de trasfondos: "el fondo del movimiento concreto es el mundo dado, el fondo del movimiento abstracto es, por el contrario, construido" (pp. 127-128), fantaseado.

La intencionalidad es siempre una proyección que va más allá de sí misma, que se dirige hacia un objeto. Si llamamos intencional a la relación que existe entre nuestro ser orgánico, animal, y los objetos de su mundo circundante es porque, como afirmó Husserl (1992), 'estamos 'dirigidos' a estos, nuestra 'intención' va hacia ellos” (p. 39). Así, todo percibir y todo efectuar es una proyección, un salir de sí o dirigirse hacia algo. "La función normal que posibilita el movimiento abstracto es una función de 'proyección' por la que el sujeto del movimiento reserva delante de sí un espacio libre" (Merleau-Ponty, 1985, p. 128) instituido intencionalmente. Fenomenológicamente, el mundo circundante del animal está entonces constituido por la articulación entre los cuerpos orgánicos y los otros cuerpos u objetos de los que se hace presa a través de intencionalidades presentes y ausentes.

Toda percepción es percepción de algo, y toda acción es acción dirigida hacia algo; todo comportamiento es teleológico, está dirigido hacia un objeto. Si existe una "estructura primigenia teleológico-tendencial" (Husserl, 1992, p. 12) en la fenomenología, es porque todo acto intencional se dirige a un fin u objetivo $^{7}$. No se trata de dar al comportamiento animal el supuesto de una finalidad

7 En "El artículo de la Enciclopedia Británica", Husserl (1992) menciona una "estructura primigenia teleológico-tendencial” de la fenomenología como autoexamen de la racionalidad de la humanidad trascendental al servicio de la producción de una praxis de la libertad. Según esto, la teleología estaría abocada a resolver todas las antítesis producidas por la filosofía escrita hasta entonces. Nosotros le damos la vuelta a esta expresión husserliana para explicitar que cualquier acto intencional tiene una estructura teleológica en cuanto siempre tiende y es guiada a la constitución de un objeto. 
absoluta en términos teológicos que, desde el exterior, determina planes y propósitos. Tenemos que reconocer el comportamiento como proyecto del mundo, como destinado a un mundo que ni abarca ni posee en su totalidad, pero hacia el cual no deja de estar orientado.

Se objetará que toda intencionalidad está esencialmente vinculada a la conciencia y que, por lo tanto, resulta complejo emplear este concepto para referirnos al hacer de formas de vida sin autoconciencia. $\mathrm{Al}$ respecto, es menester recordar que, incluso para Husserl, no toda intencionalidad puede ser tenida como un modo más de la autoconciencia. En palabras suyas:

En general, oponer esa mi esencia propia a alguna otra cosa, o el hecho de que yo, el yo que soy, pueda llegar a ser consciente de algo que me es extraño, de otro que no soy yo, presupone, por tanto, que no todos los modos de conciencia que me son propios pertenecen al círculo de aquellos que son modi de mi autoconciencia (Husserl, 2009b, p. 140).

Este es el caso de la percepción y del comportamiento, cuya estructura remite la atención a algo que se presenta de suyo como otro, y que en esta medida la trasciende. Podemos distinguir una intencionalidad totalmente activa, como toma de posición voluntaria que propone variaciones libres, de una intencionalidad operante (fungierende Intentionalität) que constituye un mundo natural y vivido a medio camino entre los movimientos concretos dirigidos al ser presente y la apertura del movimiento abstracto relacionado con el no $\operatorname{ser}^{8}$. En las lecciones sobre el estudio del comportamiento recogidas en las notas de clase que M. Merleau-Ponty dedicó a la filosofía de la naturaleza entre 1957 y $1960^{9}$ (1995, p. 220), el filósofo francés se refiere al trabajo de Uexküll como aquel momento del pensamiento biológico en el que la actividad comportamental orientada hacia el mundo circundante es asumida como un tipo particular de intencionalidad que comienza antes de la invención de la conciencia tética. Siguiendo este argumento, la conciencia sería un modo de la intencionalidad que en principio surgiría o aparecería instituido como un tipo de comportamiento. Si esto es así, entonces

\footnotetext{
8 Véase E. Husserl, Ideas II (2014) y M. Merleau-Ponty, Fenomenología de la percepción (1985).

9 Véase "L'étude du comportement animal" en Merleau-Ponty, 1995.
} 
todo lo que se comporta lo hace intencionalmente. Hay diferentes niveles de intencionalidad en los que se exponen diferentes grados de actividad o pasividad, distinción que implica, a su vez, considerar múltiples grados en los que la conciencia está involucrada en la constitución de las vivencias. En los grados superiores de la actividad encontramos la invención y la creación, mientras que en los inferiores podemos hallar los hábitos o los reflejos, por ejemplo.

\section{La intencionalidad en la conformidad a plan (Planmäßigkeit)}

De LA RELACIÓn INTENCional entre maquinistas y los objetos con los que estos interactúan podemos concluir que entre unos y otros existe una relación de motivación, imbricación y cooriginariedad. El maquinista siempre está volcado sobre algo que está más allá de sí mismo, hacia la trascendencia de los objetos de sus intencionalidades. Entre el aquí del cuerpo animal y el allí del objeto del que este hace presa hay una relación diádica: no hay aquí sin allí y viceversa; no hay saber incorporado del propio cuerpo sin saber de la diferencia respecto de aquello ahí contrapuesto. Si decidimos llamar mundo circundante (Umwelt) a ese espacio que se abre entre punto y contrapunto, entre aquí y allí, entonces el origen del organismo y la apertura de su mundo son fenómenos conjuntos. Pero ¿qué implica esta relación de codependencia entre sujeto y objeto en el desarrollo ontegenético de los seres vivos? Como vimos, las reglas de origen y de funcionamiento propuestas por Uexküll comparten el mismo telos. Lo que ahora requiere una explicación más completa es cómo la melodía de formación se relaciona con la melodía funcional más allá de la distinción implícita en el paso de una a otra tras alcanzar el punto crítico.

Siguiendo a Uexküll (2014a), "si se lo observa de cerca, esta regla [la melodía funcional] es -como regente del organismo que trabaja- el auténtico fin hacia el que tiende la melodía de formación" (p. 103). En este sentido, la melodía de formación que sigue una finalidad (Zielstrebigkeit) que se desenvuelve temporalmente es impulsada por una formación que conecta plenamente al organismo con su mundo. La armonía entre cuerpo y mundo, el ajustamiento entre el organismo y espacio que habita, es el fin viviente del impulso formador. Para el etólogo, como para el fenomenólogo, una existencia es siempre un ser in-mundo, o mejor, ser-del-mundo. Entre ambos hay una relación de copertenencia que Uexküll cree 
es constituida teleológicamente. La conformidad a plan (Planmäßigkeit) que se manifiesta en el ajustamiento une todas las relaciones existentes entre organismo y mundo en un cambio continuo, pero reglado. Más que una potencia conformadora de cuerpos, la conformidad a plan es una fuerza formadora de relaciones entre sujetos y mundos. Si la melodía de formación se encontraba bajo el signo de un telos que se alcanza en el horizonte temporal, la melodía funcional se haya dirigida por un polo exterior al cuerpo orgánico con el que se establecen relaciones recíprocas y complementarias.

Comparando la teleología de la Crítica del discernimiento con la teleología expuesta en la conformidad a plan uexkülliana, encontramos que esta última nos invita a pensar en una suerte de metateleología, en tanto que supera los límites del concepto de organismo como fin en sí mismo. Recordemos que, según la "Crítica del juicio teleológico", juzgamos los organismos vivos como fines de la naturaleza cuando estos son causa y efecto de sí mismos. De ahí los dos requisitos que un objeto debe satisfacer para ser considerado una criatura organizada: (1) cada una de sus partes solo puede ser posible gracias a su relación con el todo y (2) cada una de sus partes debe estar enlazada en la unidad con el todo, de suerte que cada una de ellas sea causa y efecto de su propia forma ( $K U \mathrm{Ak}$. V373). Luego un fin de la naturaleza es aquel en el que cada parte es pensada como órgano que solo existe en virtud del todo. Por esta razón, Kant considera que el cuerpo orgánico solo puede ser comprendido según su posibilidad interna, con lo que toda referencia a las condiciones de posibilidad externas de la vida queda excluida. Evidentemente, el problema con la apuesta kantiana es su fijación con la forma del organismo, fijación que no le permitió ver el entramado vital que se teje alrededor de un cuerpo. Allí donde la miopía trascendental de Kant pone la forma del individuo como unidad de juicio, Uexküll propone la esfera compuesta por el organismo y sus objetos de experiencia, es decir, el mundo circundante, como unidad mínima de estudio y de sentido.

La naturaleza hila lazos invisibles que conectan los fenómenos exteriores y los cuerpos vivos, otorgándoles propiedades complementarias a ambos. El trato con los objetos queda incluido en el círculo de la conformidad a plan del organismo, pues no es posible pensar a este último sin el ámbito en el que vive. Cada símbolo de significación de un sujeto es al mismo tiempo un motivo de significación para la estructura del propio sujeto (Uexküll, 2010). El mundo circundante es 
constituido por el sujeto como espacio de sentido, y el sujeto es una estructura que expresa especularmente su mundo. Lo anterior resulta impensable a la luz de las estrechas posibilidades abiertas por Kant para la consideración teleológica de los seres naturales. Por un lado, porque suponer una finalidad exterior perteneciente a los componentes del medio implicaría aceptar una suerte de intención de la materia, con lo que admitiríamos la existencia de un ingenio de los elementos que intervienen en la modelación de los cuerpos de acuerdo con un propósito. Kant considera que las cosas que no poseen ninguna finalidad en sí mismas, como la tierra, el aire, el agua, etc., solo pueden ser objetos conformes a fines externos a sí mismos y en relación con seres organizados (KU Ak. V425); por otro lado, para el filósofo de Königsberg, siempre que pensamos en una finalidad exterior al organismo buscamos una condición más allá, tanto del organismo, como del sistema físico-teleológico general, esto es, pensamos en una incondicionalidad o fin final exterior al ámbito natural (KU Ak.V378), recayendo así en la metafísica. Así pues, Kant cree que, si un organismo ha de tener alguna finalidad externa, esta debe ser encontrada únicamente en su relación con otro ser vivo distinto a él; el ejemplo paradigmático es la red o cadena alimenticia ${ }^{10}$. Ciertamente, desde esta perspectiva, la regla de formación y la regla de función no solo son incomparables, sino que la segunda no juega rol alguno en el desenvolvimiento de la primera. Por el contrario, la biología teórica uexkülliana no puede disociar la regla de formación de la regla de funcionamiento. El origen de un organismo, y de cualquiera de sus órganos, está regido desde el principio por una finalidad tanto interior como exterior.

La intencionalidad es el "medio en el medio" que vincula organismo y mundo, aparentemente opuestos. Entre el campo de sentido y una existencia en el mundo hay una relación intencional que es vivida, una causalidad bidireccional que tercia entre ambos. Empero, los hilos que enlazan al organismo con el mundo están hechos de goma, no son rígidos. Esto quiere decir que los vínculos perceptivos y efectivos entre animal y los objetos que encuentra en su mundo no están determinados según una forma de relación ya dada, sino que, al igual que la

10 Cuando inquirimos por la finalidad externa de un organismo preguntamos ¿para qué existe? En la Crítica del discernimiento Kant considera que solo existe una finalidad externa conectada con la finalidad interna de un organismo: la organización sexual. 
ontogénesis, comportan distintos grados de plasticidad y apertura. Podemos decir que aquí hay más un impulso formador que una forma acabada. Siempre cabe esperar cambios en las facultades plásticas tanto de órganos perceptores como efectores; por lo mismo, es posible esperar el surgimiento de hábitos nuevos. Las leyes teleológicas de la vida son indivisibles e indestructibles; y, no obstante, no están limitadas en forma ni número, pues la producción de diversidad es propia de la vida.

La conformidad a plan solo se realiza en un mundo circundante que, con todo, no está formado definitivamente, sino hasta que aparece para el organismo. Ni la estructura del organismo ni la estructura de su mundo están formadas y dadas antes de su origen simultáneo. En ambos casos, la forma es posterior al proceso de formación. Ni el sujeto ni el mundo son de suyo unidades acabadas y consumadas, son acontecimientos dinámicos en camino de ser y de hacerse mutuamente. Justamente, la biología de Uexküll comparte con la fenomenología la intención de poner en entredicho las categorías de sujeto y objeto, así como su relación de simple contraposición y autonomía. Por un lado, para Uexküll un sujeto es un organismo que se encuentra en un ámbito que le pertenece y del que hace parte, que se forma desde sí mismo a la vez que forma el espacio que le rodea. Por el otro, el mundo es tanto la condición natural de vida del sujeto, como su producto, tanto una exterioridad como una vivencia inmediata del organismo.

"La constitución de mundos de cualquier especie [...] está sujeta a las leyes de la constitución orientada" (Husserl, 2009b, p. 174) a partir de la propia corporalidad. En estos mundos hay algo constituido primordialmente, pleno y coherente, y algo constituido secundariamente, carente e incoherente. En cada existencia se manifiesta una perspectiva singular de la totalidad que, no por ello, deja de expresar cierta prodigalidad interior. Lo anterior ilumina el siguiente comentario de Uexküll:

Todas las leyes técnicas de la naturaleza viviente se manifiestan en la forma de unidades subjetivas que forman siempre un nuevo centro del mundo que, a su vez, presenta relaciones -a veces más ricas, a veces más pobres- con su mundo circundante, pero siempre representa una perfección acabada en sí misma (2014a p. 147). 
El naturalista que observa ha de aprender a juzgar las interacciones del animal con su mundo de acuerdo con el tipo de correspondencia inmanente que se establece entre estos dos, no según la perspectiva que él tiene respecto de un mundo que nunca llegará a ser más que exterior y objetivamente extraño.

\section{La apertura afectiva a la multiplicidad de mundos circundantes}

La Ley de Johannes P. Müller afirma que el tipo de sensación que sigue a un estímulo está directamente relacionado con el modo específico en que el órgano sensitivo está organizado y depende de él (Rachlin, 2005). Para Müller, el sistema nervioso encarnaba las formas de la sensibilidad y las categorías del entendimiento. Este idealismo fisiológico implica una inversión (Umkehrung) en la que las formas de la sensibilidad son puestas, no por un sujeto trascendental, sino por cierto aparato biológico propio de cada especie. A partir de ello, Uexküll concluyó que la forma del mundo circundante, y de los objetos que lo constituyen, se encuentra inscrita y bosquejada en el cuerpo del organismo. Los objetos son producto de la información de una anatomía subjetiva que constituye la forma (Gestalt) y el contenido de los fenómenos. Uexküll llama forma estructural (Bauplan) a esta organización que levanta el mundo circundante de los seres vivos de acuerdo con una organización anímico-corporal concreta. En otras palabras: el cuerpo de cada organismo dibuja los planos arquitectónicos de su mundo. Por esto "las relaciones entre objeto y sujeto se basan siempre en efectos que parten del objeto y se encuentran con los órganos sensoriales del sujeto" (Uexküll, 2014a, p. 40). Los órganos sensoriales de los animales son apropiados para recibir un grupo determinado de efectos que son emitidos por ciertas características (Merkmale) de los objetos.

Desde un principio se supone que, cuando se le presenta un mismo objeto a dos sujetos que poseen distintos órganos sensoriales, las propiedades del objeto que actúan como características deben ser distintas para cada uno de los sujetos. Por lo tanto, la experiencia de ambos, aunque se refieran al mismo objeto, necesariamente se contradice, porque el objeto posee distintas propiedades para cada sujeto (Uexküll, 2014b, p. 40).

Según lo anterior, la función de los órganos es, primero, poner al alcance del sujeto un grupo determinado de estímulos; segundo, inhibir u opacar los efectos 
restantes. Esto llevó a Martin Heidegger (2007) a determinar la diferencia en las esencias del animal y el hombre de la siguiente manera: "1) la piedra no tiene mundo [Weltlos]; 2) el animal es pobre de mundo [Weltarm]; 3) el hombre configura mundo [Weltbildend]" (p. 227). Partiendo de los textos de Uexküll, Heidegger apunta que el animal es pobre de mundo pues su modo de accesibilidad a aquello con lo que guarda una relación en tanto que viviente carece de posibilidades: el animal está atrapado en los círculos funcionales definidos por sus características corporales. La lectura que Heidegger hace de Uexküll toma el arco reflejo como modo de comportamiento (Benehmen) por excelencia. De ahí que el autor de Los conceptos fundamentales de la metafisica entienda el perturbamiento (Benommenheit) como modo de ser propio del animal. ¿Qué significa esto? Básicamente, que el animal no obra libremente, sino que sus comportamientos siempre son respuestas condicionadas por un estímulo. El organismo es capaz "de...", pero sus capacidades siempre salen al encuentro del mundo condicionadamente. Los objetos imponen su sentido al animal como una solicitud que demanda una respuesta inmediata. La animalidad descrita por Heidegger está entonces abierta a un mundo circundante tan reducido que más vale decir que está encapsulada en él.

Ahora bien; que el mundo sea configurado por el hombre revela, por el contrario, que la totalidad del ente no solo se le manifiesta a este como algo concreto y dado, sino que el ente puede manifestársele de distintas maneras. De acuerdo con Heidegger, la excepcionalidad de esta manifestabilidad es posible gracias a los temples de ánimo fundamentales que atraviesan toda manifestación del mundo para el Dasein. La configuración del mundo -que algo se dé en tanto que es algo para el hombre- está atravesada por el posible develamiento del ser del

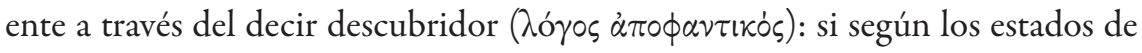
ánimo el mundo se nos aparece como una cosa o la otra, es a través del hacer-ver (àmó $\phi \alpha \sigma ı \zeta)$ que desocultamos o explicitamos aquello que permanecía implícito en la afectividad. Luego, la configuración del mundo humano parece estar ligada irremediablemente tanto a la afectividad como al lenguaje. Si quisiéramos superar semejante lectura heideggeriana y mostrar en qué medida la animalidad también es configuradora de mundo (Weltbildend), tendríamos que exponer en qué sentido esta no es presa de sus círculos funcionales, cómo la afectividad atraviesa la relación del mundo con el animal, y gracias a qué intencionalidades y 
potencialidades el animal produce, se representa y hace destacar lo que abarca y que lo rodea.

En un pasaje oscuro y complejo de Los conceptos fundamentales de la metafisica en el que parte de Aristóteles para analizar los elementos estructurales del decir descubridor, Heidegger (2007) señala que

si todo $\lambda$ ó ${ }^{\circ} \circ$ verdadero y todo $\lambda$ ó $\gamma \circ \varsigma$ falso puede ser o bien $\kappa \alpha \tau \alpha \phi \alpha \sigma \iota \varsigma$ o bien

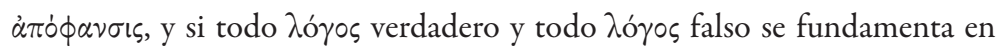

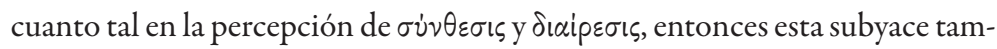

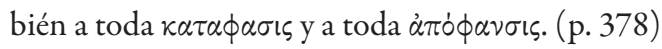

Lo que algo es puede ser develado gracias a que podemos distinguirlo de aquello que no es. Así, el develamiento del ser que acontece en el decir descubridor está fundado en la capacidad de la percepción para unir y separar, para sintetizar y dividir, para articular y distinguir. ¿Qué nos impide afirmar lo mismo del modo en que perciben otros organismos? Aunque la corporalidad del organismo expone unas leyes generales que bosquejan las posibilidades y límites de la experiencia, estos marcos no son rígidos sino flexibles. Aquí, como en el proceso de morfogénesis del individuo, podemos hablar de una epigénesis del mundo: el mundo circundante del animal es un acontecimiento que se va formando desde lo general, su estructura espaciotemporal, hasta el detalle de sus particularidades.

En el estar dirigido a un más allá de sí mismo en la percepción, el animal no solo tiende, sobre todo atiende. El mundo circundante no se da como un ámbito de homogeneidad en el que la única diferencia perceptiva es marcada por la intensidad o amplitud de los estímulos, como si el animal solo pudiera escuchar lo más ruidoso, o ver lo inmediatamente más cercano. Como expresó adecuadamente Merleau-Ponty (1985, p. 50), la atención supone, en principio, una transformación, una nueva manera de estar presente frente a los objetos. La percepción sintetiza unidades objetuales de experiencia al tiempo que separa y

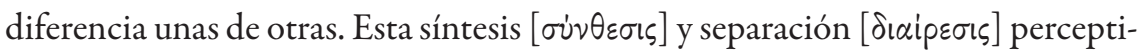
vas develan o hacen-ver [ $\dot{\alpha} \pi \dot{\phi} \phi \alpha \nu \sigma \iota \zeta]$ porque velan $[\kappa \alpha \tau \alpha \phi \alpha \sigma \iota \zeta]$ ciertos elementos del mundo. Entendemos entonces que, por ser una actividad, la atención perceptiva es una fuerza o impulso formador (Bildungstrieb) y no un transformador receptivo de datos con formas (Gestalten) en contenidos determinados. Al igual que el impulso formador que rige la ontogénesis, la atención está en relación con 
un fin u objetivo, el objeto de atención. Prestar atención no es solamente clarificar algunos elementos preexistentes del mundo circundante, es realizar en él una nueva articulación, una nueva configuración. La atención es "la construcción activa de un objeto nuevo que explicita y tematiza lo que hasta entonces solamente se ofrecía a título de horizonte indeterminado" (Merleau-Ponty, 1985, p. 52).

Que un objeto aparezca con preeminencia, que la atención perceptiva haga aparecer un extraordinario como figura del fondo ordinario, no puede entenderse sin el deseo, el amor, la furia o la rabia que los hace emerger. No hay relación intencional que se dé sin afecto. El mundo del animal es considerado pobre y limitado solo si se cree que su relación con el organismo es desinteresada y sin compromiso. La afectividad anima el mundo, convierte en cuerpo de atracción o repelencia lo que desde una mirada científica son meros objetos o signos. En este sentido, la afectividad es configuradora de mundo (Weltbildend), es una potencia poiética. Heidegger falla al partir del arco reflejo como modo por excelencia de todo hacer animal, lo que le impide reconocer en el animal un estado de ánimo que no sea el perturbamiento (Benommenheit).

El producto de la afectividad, la imagen poética según Bachelard (1997, p. 10), es esencialmente variable. Con esto queremos decir que las coloraciones específicas y exclusivas de una forma de experimentar el mundo circundante no cuentan dentro de las estructuras constitutivas de la experiencia formal, aquello común a todos los individuos de una misma especie determinado por su corporalidad general. Por el contrario, modulan los vínculos entre los sujetos particulares y sus objetos singulares. ¿Cuántas variaciones puede sufrir la forma del mundo para un organismo? Tantas como alteraciones en sus estados de ánimo puedan experimentar estas existencias. Uexküll describe cómo la imagen del mundo del cangrejo ermitaño posee un esquema formal bastante sencillo: todo objeto de forma cilíndrica o cónica es percibido como marcador de sentido. Sin embargo, la descripción formal es insuficiente si preguntamos por qué el mismo objeto cambia de significado para este animal. "Para representar las cosas provistas de una relevancia vital en el mundo circundante de un animal, le prestamos a la imagen perceptual que proveen los sentidos un tono efectual, para captar íntegramente su significación" (Uexküll, 2016, p. 105). Aunque no realiza un análisis propiamente fenomenológico, Uexküll (2016, p. 102) reconoce que el cambio de significado de un objeto en el mundo circundante ocurre de acuerdo 
con el estado de ánimo del organismo: según este aparezca bajo un "tono defensivo", un "tono alimenticio" o un "tono habitacional". En los tres casos el sentido del noema oscila según la noesis, rompiendo cualquier esquema fijo. Por ser objeto de múltiples intenciones, el sentido de un solo objeto se encuentra entonces abierto.

Las relaciones entre organismos y objetos se vuelven más tangibles gracias a los afectos. Comportamiento y afectividad se iluminan mutuamente. Los afectos pueden inferirse de las acciones de los animales; las prácticas cobran sentido a la luz de ellos. En palabras de Uexküll (2016): "el mundo circundante recién adquiere su admirable certidumbre para los animales que lo habitan al tomarse en cuenta los tonos efectuales" (p. 106). Karl Ernst von Baer mostró que el tiempo es distinto según las características intraorgánicas y perceptivas de cada especie, que el espacio se hace más grande conforme el cuerpo percibe un área mayor de lugar, por ejemplo. Pero por qué el tiempo de un único organismo corre con mayor o menor velocidad, o por qué un espacio objetivamente amplio puede asfixiar mientras que otro decididamente reducido puede parecer más vasto para dos individuos de la misma especie, esto solo puede encontrar una explicación en los estados de ánimo que tiñen de desazón o ilusión los mundos de los animales.

\section{Referencias}

Bachelard, G. (1997). La poética del espacio. (Trad. E. de Champourcin). México: Fondo de Cultura Económica. Baer, K. E. von. (1864). Reden gehalten in wissenschaftlichen Versammlungen und kleinere Aufsätze vermischten Inhalts. San Petersburgo: Schmitzdorff.

Buchanan, B. (2008). Ontoethologies: The Animal Enviroment of Uexküll, Heidegger, Merleau-Ponty and Deleuze. New York: State University of New York Press.

Despret, V. (2018). ¿Qué dirían los animales... si les hiciéramos las preguntas correctas? (Trad. S. Puente). Buenos Aires: Caja Negra.

Ginsborg, H. (2014). Kant on Understanding Organisms as Natural Purposes. En: The Normativity of Nature: Essays on Kant's Critique of Judgement (pp. 255-280). Oxford: Oxford University Press. 
Heidegger, M. (2007). Los conceptos fundamentales de la metafisica. Mundo, finitud y soledad. (Trad. A. Ciria). Madrid: Alianza Editorial.

Husserl, E. (1992). El artículo 'Fenomenología' de la Enciclopedia Británica. En: Invitación a la fenomenología (pp. 35-73). (Trad. A. Zirión). Barcelona: Paidós.

Husserl, E. (2009a). Investigaciones lógicas (vols. I-II). (Trads. M. G. Morente \& J. Gaos). Madrid: Alianza Editorial.

Husserl, E. (2009b). Meditaciones cartesianas. (Trad. M. A. Presas). Madrid: Tecnos.

Husserl, E. (2014). Ideas relativas a una fenomenología pura y una filosofia fenomenológica. Libro segundo: investigaciones fenomenológicas sobre la constitución. (Trad. A. Zirión Quijano). Ciudad de México: Instituto de Investigaciones Filosóficas, Fondo de Cultura Económica.

Kant, I. (2012). Crítica del discernimiento. (Trads. R. R. Aramayo \& S. Mas). Madrid: Alianza Editorial.

Lenoir, T. (1989). The Strategy of Life: Teleology and Mechanics in NineteenthCentury German Biology. Berlin: Springer-Verlag.

Merleau-Ponty, M. (1985). Fenomenología de la percepción. (Trad. J. Cabanes). Barcelona: Planeta Agostini.

Merleau-Ponty, M. (1995). La Nature. Notes cours du Collège de France. París: Éditions Du Seuil.

Rachlin, H. (2005). What Müller's Law of Specific Nerve Energies Says about the Mind. Behavior and Philosophy, 33, 41-54. https://www.jstor.org/stable/27759507

Roe, A. S. (2003). Matter, Life, and Generation: Eighteenth-Century Embryology and the Haller-Wolff Debate. Cambridge, Estados Unidos: Cambridge University Press.

Simondon, G. (2015). La individuación a la luz de las nociones de forma y de información. (Trad. P. Irés). Buenos Aires: Cactus.

von Uexküll, J. J. (1945). Ideas para una concepción biológica del mundo. (Trad. R. M. Reneiro). Madrid: Espasa-Calpe.

von Uexküll, J. J. (2010). A Theory of Meaning. (Trad. J. D. O’Neil). Minneapolis: University of Minnesota Press. 
von Uexküll, J. J. (2014a). Cartas biológicas a una dama. (Trads. T. Bartoletti \& L. C. Nicolás). Buenos Aires: Cactus.

von Uexküll, J. J. (2014b). Umwelt und Innenwelt der Tiere. Berlín: SpringerVerlag.

von Uexküll, J. J. (2016). Andanzas por los mundos circundantes de los animales y los hombres. (Trad. M. Guntin). Buenos Aires: Cactus.

von Uexküll, J. J. (2017). Theoretical Biology. (Trad. D. L. Mackinnon). Londres: Forgotten Books. 